\title{
Decolonizar la universidad
}

\author{
Eduardo Restrepo ${ }^{1}$ \\ "Las universidades han sido a la vez los talleres de la ideología y los \\ templos de la fe". \\ Immanuel Wallerstein (2006, p. 72).
}

\section{Introducción}

El título de este artículo se basa en el doble supuesto de que la universidad está colonizada y de que es posible descolonizarla. Ambos supuestos requieren ser explicitados, ya que no es tan evidente lo que implican. Tres son los planos en los cuales se puede vislumbrar la universidad colonizada. El primero de ellos, siguiendo a Foucault, hace referencia a que la universidad está colonizada por los sistemas científicos y el conocimiento experto en su pretensión de sistematicidad y de verdad. El segundo, desde la perspectiva de la modernidad/colonialidad, tiene que ver con la universidad como dispositivo privilegiado de la colonialidad del saber, con lo cual se naturaliza su eurocentrismo. Finalmente, el tercer plano hace énfasis en cómo el establecimiento universitario se encuentra inscrito en una geopolítica del conocimiento articulada como un aspecto del sistema mundo.

En el primer aparte de este ensayo se amplían estos tres planos. Algunas expresiones de estos tres planos de la colonización de la universidad son examinadas en el siguiente aparte, específicamente los discursos de la internacionalización, la productividad y la calidad que han orientado la universidad hacia un modelo empresarial y ha posicionado la imaginación burocrática insertando una serie

$1 \quad$ Doctorado en Antropología (con énfasis en Estudios Culturales), Universidad de Carolina del Norte-Chapel Hill. Maestría en Antropología, Universidad de Carolina del Norte- Chapel Hill. Antropología, Universidad de Antioquia. Profesor Asociado. Departamento de Estudios Culturales. Facultad de Ciencias Sociales. Universidad Javeriana. 
de indicadores que tienden a la normalización de las prácticas de producción de conocimiento.

Posteriormente, se plantean tres estrategias para descolonizar la universidad: la intervención genealógica, el giro decolonial y la provincialización de Europa. Estas estrategias se plantean reconociendo que la problematización y la transformación de la universidad es una ardua tarea que solo tendrá algún efecto si no se conecta con fuerzas históricas que reviertan las tendencias hacia los modelos empresariales de la universidad y la gubernametalización del conocimiento.

\section{Tres planos de colonización de la universidad}

En una de sus clases más conocidas, Michel Foucault (2001) establecía una distinción entre los saberes sometidos, los conocimientos eruditos y los sistemas científicos. Los sistemas científicos se caracterizan por una pretensión totalizante y de verdad, que subordina una serie de saberes haceres y de conocimientos de anticuario. Que unos saberes fuesen sometidos y unos conocimientos excluidos en el proceso de posicionamiento de los sistemas científicos es el resultado de una multiplicidad de luchas, aparecen como el efecto de una historicidad belicosa más que de la progresiva y teleológica aparición de la verdad.

La labor genealógica consistía en gran parte en una cartografía de estas luchas que habían sumergido en el olvido, silenciado o subsumido una serie de conocimientos y saberes haceres. De ahí que Foucault considerara las genealogías como anti-ciencias antes que como una nueva ciencia. En esta perspectiva, sin embargo, los saberes sometidos no pueden confundirse con los saberes de los sujetos sometidos, con los saberes de los sectores subalternos. Son saberes subalternizados, por supuesto; aunque no necesariamente saberes de los sometidos o subalternos.

En la universidad han sido históricamente dominantes los sistemas científicos y, en general, los conocimientos expertos. En consecuencia, los conocimientos eruditos y los saberes sometidos han estado fuera de lugar. De ahí que solo pueden ser considerados 
en el ámbito universitario cuando aparecen como "objetos" de estudio para una disciplina, cuando son diseccionados, objetivizados e incorporados por conocimientos expertos dentro de protocolos académicos establecidos. La universidad ha sido colonizada por los sistemas científicos y por sus conocimientos expertos. Y lo ha sido desde hace ya tanto tiempo que la reproducción de estos sistemas y conocimientos pareciera definir en gran parte a la universidad misma.

El segundo plano de colonización de la universidad se hace explícito desde las elaboraciones en torno a la modernidad/ colonialidad realizadas por una colectividad de académicos situados tanto en América Latina como en los Estados Unidos (Escobar 2003; Restrepo \& Rojas 2010). Desde esta perspectiva, se puede argumentar que el aparato universitario hace parte de un dispositivo epistémico mucho más general que ha sido denominado colonialidad del saber.

El término de colonialidad del saber se ha derivado del de colonialidad del poder, sugerido por el sociólogo peruano Aníbal Quijano (2000) hace unos veinte años. La colonialidad no es equivalente a colonialismo. La colonialidad sería la lógica que subyace al colonialismo, pero que se mantendría incluso hasta nuestros días, ya que se concibe como el lado oscuro e inmanente de la modernidad. Siguiendo una línea de argumentación que encuentra en Aimé Césaire (2006) un referente importante, no hay modernidad sin colonialismo, la civilización moderna occidental no se puede separar de la violencia colonial sobre la que se ha edificado. La colonialidad del poder refiere al patrón de poder global que surge con el sistema mundo moderno, y que, asociado a una clasificación racial, ha permitido el control y explotación de fuerza de trabajo, riquezas y territorios a lo largo del planeta en aras de la emergencia y consolidación del capitalismo (Mignolo, 2007).

La colonialidad del saber refiere a la dimensión epistémica de la colonialidad del poder. La colonialidad del saber es constituida por un patrón de clasificación y jerarquización global de los conocimientos, donde unos aparecen como la encarnación del conocimiento auténtico y relevante, mientras que otros conocimientos son expropiados, inferiorizados y silenciados, a tal punto que dejan de ser conocimientos para aparecer como ignorancias, supersticiones. 
Los conocimientos son clasificados a partir del paradigma teológico primero y del científico después, desestimando aquellas modalidades de conocimiento que escapan a la legibilidad y apropiabilidad de estos paradigmas. Esta clasificación implica no sólo la ecuación entre conocimiento verdadero y conocimiento europeo occidental encarnado en sus sistemas religiosos, filosóficos y científicos, sino también la inferiorización cuando no borramiento de otras modalidades de conocer de "los otros" de Europa:

[...] la colonialidad del saber, [debe ser] entendida como la represión de otras formas de producción del conocimiento (que no sean blancas, europeas y 'científicas'), elevando una perspectiva eurocéntrica del conocimiento y negando el legado intelectual de los pueblos indígenas y negros, reduciéndolos como primitivos a partir de la categoría básica y natural de raza (Walsh 2005, p 19).

Aunque la colonialidad del saber también subordina unos saberes-haceres y conocimientos en nombre de la ciencia y el conocimiento experto (como vimos unos párrafos antes en el análisis de Foucault), el énfasis de la perspectiva de la colonialidad del saber radica en evidenciar el eurocentrismo racializado del conocimiento teológico o científico que ha sido constitutivo de Occidente. De ahí que el aparato universitario occidental ha estado estrechamente ligado a la colonialidad del saber.

El tercer plano de colonización de la universidad está relacionado con la geopolítica del conocimiento. La geopolítica del conocimiento es una perspectiva que se pregunta por la situacionalidad del conocimiento, pero no sólo en su producción, sino también en su circulación y en sus apropiaciones. Boaventura de Sousa Santos (2009) considera que la perspectiva de la geopolítica del conocimiento supone: "[...] quién produce el conocimiento, en qué contexto lo produce y para quién lo produce" (p. 340).

Desde la geopolítica del conocimiento es imposible la existencia de un conocimiento des-subjetivado, descorporalizado. El conocimiento está siempre anclado y marcado por los sujetos que lo producen, independientemente de sus capacidades reflexivas 
para comprender y cartografiar estas improntas. Las historias y trayectorias de estos sujetos (las cuales no sólo operan en el registro de lo mental sino que se han hecho cuerpos) troquelan de las más diversas maneras, no sólo el conocimiento como resultado, sino las posibilidades e imposibilidades mismas del conocer. Ciertas experiencias o condiciones de los sujetos concretos invisten de maneras difíciles de separar (cuando no incluso de identificar) la producción misma del conocimiento.

También es pertinente preguntarse por el contexto, es decir, por los lugares institucionales, sociales y geo-históricos desde dónde se produce el conocimiento. Boaventura de Sousa Santos comparte con muchos otros autores y corrientes teóricas la idea de que el lugar importa en cuanto a lo que se produce. Por lugar se entiende las ubicaciones institucionales (establecimientos metropolitanos/ periféricos, académicas/no académicas, gubernamentales/ no gubernamentales, etc.), las sociales (raciales, etnizadas, culturalizadas, sexuales, generacionales, de clase, de género, etc.) y geo-históricas (occidente/ el resto, civilización/barbarie-salvajismo, desarrollo/ subdesarrollo, formaciones nacionales, regionales y locales, etc.).

Esta densa filigrana de marcaciones hace que nos encontremos siempre ante conocimientos situados de múltiples maneras. Las improntas de lugar, en su irreductible historicidad, hacen de la producción del conocimiento algo bien distinto de la idea de conocimientos puros y universales, que estarían más allá de las belicosidades y avatares de sus tiempos. Finalmente, esta perspectiva geopolítica del conocimiento supone la pregunta de para quién se lo produce. Este para quién es, en últimas, una pregunta por el para qué del conocimiento e, hilando aún más delgado, una preocupación ética fundamental de con quién se produce tal conocimiento.

Desde esta perspectiva, el sistema mundo debe ser pensado no sólo en los registros más obvios como el de un orden económico o político mundial, sino que también pasa por aspectos más sutiles como las configuraciones culturales o, todavía más específicamente, el establecimiento universitario. Desde el análisis del sistema mundo, estos campos suponen una densa red de relaciones de flujos y jerarquías, donde unos establecimientos académicos de ciertos 
lugares del mundo se encuentran mejor posicionados que otros para definir los términos y las condiciones de las discusiones al interior de cada campo. ${ }^{2}$

Este tipo de análisis de la geopolítica del conocimiento permite examinar el tercer plano de colonización de la universidad al evidenciar que predominan en la universidad unas ideas de los conocimientos científicos y expertos como si fuesen descorporizados y descontextuados, mientas que las respuestas del para quién y para qué se produce el conocimiento se orientan cada vez más explícitamente a la satisfacción de las demandas del mercado y del sector empresarial.

\section{La universidad corporación y la imaginación burocrática}

Al menos en Colombia, en las tres últimas décadas el sistema universitario se ha orientado hacia un modelo empresarial, donde los discursos de la productividad, la calidad y la internacionalización se han ido posicionando sin mayores cuestionamientos. El creciente papel protagónico de una imaginación burocrática asociada a este modelo es también otro de los rasgos que han permeando, con mayor o menor fuerza, la labor cotidiana en las diferentes universidades.

Hoy en el establecimiento académico y en los practicantes de las ciencias sociales y humanidades predomina una agenda muy distinta. Con las transformaciones de las políticas de ciencia y tecnología en Colombia y del campo universitario en general se han posicionado una concepción de investigación que hipervalora las publicaciones (sobre todo de artículos en revistas indexadas, ojalá en inglés) y unas nociones bien específicas de productividad y calidad que se han vinculado con las carreras y salarios de los académicos. Esto ha propiciado unos ritmos y propósitos de la investigación que suelen darle la espalda a la producción de conocimiento crítico en general y a la vinculación sustantiva de los académicos con procesos y movilizaciones sociales de los sectores subalternizados.

2 En tal sentido, la categoría de sistema mundo ha sido utilizada para entender las diferencias y asimetrías entre las diferentes tradiciones antropológicas del mundo. Ver, por ejemplo, a Gustavo Lins Ribeiro y Arturo Escobar (2008) y al antropólogo japonés Takami Kuwayama (2004). 
En las tres últimas décadas el sistema universitario se ha orientado hacia un modelo empresarial, donde los discursos de la productividad y la calidad se han ido posicionando sin mayores cuestionamientos. El creciente papel protagónico de una imaginación burocrática asociada a este modelo es también otro de los rasgos que han permeando, con mayor o menor fuerza, la labor cotidiana en las diferentes universidades.

Si bien las universidades privadas en nuestros países se han concebido desde la lógica empresarial de la rentabilidad, lo que ha cambiado en las últimas dos décadas es la generalización del modelo gerencial en universidades públicas y privadas, donde se imponen cada vez más las preocupaciones por la rentabilidad de los programas ofrecidos y la transformación de los estudiantes en clientes. Las burocracias universitarias han ido extendiendo su accionar con la creciente regulación de las prácticas académicas a través de la imposición de una filigrana de procedimientos y formatos a los que cada vez se hace más difícil escapar y que tienden a consumir más tiempo y dedicación.

Así, lainvestigaciónse haconvertidoenunaactividad fuertemente regulada y estructurada por demandas de las burocracias académicas. Para solicitar recursos o descarga de tiempo, el investigador se ve compelido a operar dentro de una serie de requerimientos de ritmos y productos fijados por las burocracias universitarias. Las consultorías se imponen cada vez más como el escenario de la investigación, lo que la subsume a los intereses de quienes contratan.

Las metodologías de investigación invasivas y apuradas, encorsetadas en limitados cronogramas de proyectos que deben entregar resultados visibles y valorados por la burocracia académica o estatal, son las que predominan hoy en el grueso de las ciencias sociales y humanidades. Encuestas, entrevistas, grupos focales y talleres realizados con celeridad son técnicas de investigación usuales para darle cierto fundamento empírico a los cientos de artículos y libros que engrosan los Gruplac's y Cvlac's de los grupos de investigación e investigadores en el país. Aunque algunos mantienen retóricas críticas y enuncian sus "compromisos con las comunidades", en sus prácticas suelen operar dócilmente en los marcos definidas 
por las burocracias de sus universidades y por las expectativas del establecimiento académico convencional.

El modelo empresarial y la imaginación burocrática operan y refuerzan los tres planos de colonización de la universidad indicados. La pretensión de totalidad y de verdad de los sistemas científicos y conocimientos expertos, el reforzamiento del eurocentrismo y el desconocimiento de las relaciones de poder del sistema mundo, definen en gran parte lo que caracteriza al sistema universitario presente al menos en un país periférico como Colombia. De ahí que la deseabilidad y posibilidad de descolonizar la universidad requiere de una adecuada comprensión de cómo se encuentran articulados estos tres planos de colonización y cuáles son sus expresiones concretas.

Estas regularizaciones sobre las labores de los docentes e investigadores en las universidades evidencian una transformación sustancial en la concepción misma de la universidad. De una idea de universidad articulada por la función ético y política humanista de devenir en instancia para la reflexión crítica y autónoma de la sociedad, se ha ido naturalizando una noción de universidad orientada por una racionalidad instrumental tendiente a producir los tecnócratas y expertos que requiere el mercado y el estado. El modelo gerencial imperante es la punta del iceberg de los profundos cambios que se ha suscitado en la universidad, incluso en aquellas que aún se siguen considerando como públicas. ${ }^{3}$

La apuesta por la internacionalización es quizás una de las expresiones más obvias de la nueva alquimia discursiva. Internacionalización es una noción que circula con frecuencia en el ámbito universitario y en las políticas de ciencia y tecnología agenciadas por la entidad gubernamental (Colciencias ${ }^{4}$ ) para referirse

3 El proceso de privatización de las universidades públicas no hay que entenderlo simplemente desde la perspectiva de donde provienen los recursos para su funcionamiento y quiénes pueden acceder a sus programas. También hay que considerar el modelo mismo de universidad, del para qué, el cómo y el hacia dónde que se expresa en prácticas como el posicionamiento del modelo gerencial comentado.

4 Departamento administrativo de ciencia, tecnología e innovación (CTeI) que depende de la presidencia de la república y lidera el sistema nacional de cien- 
a una deseada e inevitable articulación de la "comunidad académica nacional" con la global. Deseada articulación porque supone una suerte de "elevación" de la "calidad" de la ciencia y tecnología del país a unos 'niveles superiores' que se asumen son los que existen en el "escenario internacional". Esta 'elevación' se considera consecuencia de una mayor exposición y visibilidad de la ciencia y tecnología producida en el país a los estándares de la "comunidad internacional".

También se asume la internacionalización como inevitable porque se la considera como un aspecto de la ineludible globalización a la que el país se enfrenta. Desde esta perspectiva, ante el avance de la globalización supuestamente no hay otra alternativa que la de articularse "eficientemente" a la "comunidad global". Entendida de esta manera, la internacionalización se asocia fácilmente a las predicas celebratorias de la globalización. Estas predicas constituyen, desde una aparente neutralidad, narrativas teleológicas que presentan como necesario lo que es contingente e histórico obliterando otros principios de inteligibilidad del presente (Trouillot, 2011).

Ahora bien, esto no es simplemente una inocua narrativa que embruja la imaginación de la burocracia de la ciencia y la tecnología, sino que en nombre de la internacionalización así concebida se adelanta una agresiva política de normalización de las prácticas académicas en el país. La producción y circulación del conocimiento académico han sido objeto de toda una gubernamentalización que esencialmente las reduce a una serie de indicadores y dispositivos de captura. Indicadores de ciencia y tecnología, indexaciones de las revistas, formalización de grupos de investigación y fijación de la producción de los individuos en hojas de vida estandarizadas son algunas de las intervenciones más obvias que desde la entidad gubernamental se adelantan cada vez con mayor ímpetu.

Dispositivos de captura como las plataformas en formato electrónico para registrar las trayectorias y productos de los investigadores, de los grupos de investigación y de sus proyectos. El malestar existente entre algunos académicos (la mayoría pertenecientes a las ciencias sociales y humanidades) sobre los

cia, tecnología e innovación de Colombia. 
criterios y procedimientos de estas políticas de normalización no se ha consolidado en un movimiento de crítica visible y consistente; en gran parte porque dependen de instituciones (universidades, institutos, Ong's) que se han plegado sin mayor examen a estas políticas.

Estas políticas de ciencia y tecnología han ido ganando terreno articulándose con otras tendencias para ir profundizando las normalizaciones indicadas en los profesores e investigadores adscritos a las universidades. Como lo indica Mato (2002), esto no parece ser una situación extraordinaria de Colombia:

Este es que en las últimas dos décadas han venido ganado terreno en América Latina ciertos discursos 'modernizadores' de 'la ciencia' y de las universidades que desde gobiernos y medios universitarios procuran normar, delimitar y controlar las prácticas intelectuales en términos de productividades, medidas estas por indicadores tales como cantidad de publicaciones en revistas académicas 'arbitradas', especialmente de circulación internacional; cantidad de citas de sus obras hechas por sus colegas; etc. Para ello los actores que promueven esos discursos han instituido ciertos sistemas llamados de 'estímulo de la investigación' (hasta donde sé, al menos en Argentina, Brasil, Colombia, México y Venezuela), a través de los cuales se distribuyen dineros en relación precisamente a tales tipos de indicadores. Estos reconocimientos fortalecen una idea de 'investigación' que se pretende objetiva y avalorativa, y que sin duda es marcadamente academicista (p. 22).

Al menos en Colombia, esos discursos de la internacionalización, de la calidad y la productividad se han asociado en el campo de las ciencias sociales y humanidades al doble movimiento de un auge en la creación de postgrados (maestrías y doctorados) y al socavamiento de los pregrados existentes (lo que en otros países denominan las licenciaturas o carreras). 
También se ha constatado la explosión de la aparición de pequeñas universidades con propósitos abiertamente empresariales, centradas en cierto tipo pregrados o postgrados más técnicos y con demanda del mercado. Incluso en las universidades públicas más destacadas del país, a la sombra de los discursos de la internacionalización, de la calidad y de la productividad, se han ido consolidando nociones de eficiencia que las acercan al modelo de la universidad-negocio.

\section{Hacia la descolonización de la universidad}

Los tres planos de la colonización de la universidad (por los sistemas científicos y conocimiento experto, por el eurocentrismo de la colonialidad del saber, y por las relaciones de poder asociadas a la geopolítica del conocimiento) constituyen los escenarios que requieren ser tomados en consideración en una propuesta de descolonización de la universidad. Seguramente no son los únicos, pero sin duda son de los más importantes para transformar el modelo dominante de universidad. Ahora bien, en correspondencia con cada uno de estos tres planos, se pueden identificar tres estrategias de la descolonización: la interrupción genealógica, el giro decolonial y la provincialización de Europa.

Como estrategia para la descolonización de la universidad, la interrupción genealógica permite evidenciar cómo hemos llegado a ser lo que somos a partir de ciertas políticas de la verdad al trazar las luchas de las que han emergido los sistemas científicos y los conocimientos expertos sepultando unos conocimientos históricos y saberes "menores". Desde esta perspectiva, la interrupción genealógica es una estrategia de historización radical de los múltiples efectos de las pretensiones de verdad y de totalidad de las ciencias y expertos.

Alcuestionarsus arrogancias epistémicas, se abrirían condiciones de posibilidad para que tengan lugar en la universidad multiplicidad de saberes sometidos sin que se deban subsumir a objetos de estudio de las ciencias establecidas y a sus protocolos académicos. Posibilitar la circulación en la universidad de estos saberes irreverentes que tengan efectos desencajantes en las totalizaciones disciplinadas de las ciencias y conocimientos expertos. La descolonización de la 
dominancia de los sistemas científicos y de conocimientos expertos en la universidad, no significa que la propuesta sea la simple eliminación de estos sistemas y conocimientos para colocar en su lugar los saberes sometidos.

El conocimiento científico y el experto constituirían unas modalidades de saber, que no estarían por encima, al margen o en anterioridad de las relaciones de poder. No obstante, esto ha sido leído erráticamente como si en el planteamiento foucaultiano se estuviera argumentando una reducción del conocimiento científico y el conocimiento experto a las intencionalidades políticas de los sectores dominantes o como si todo fuese solo poder, haciendo irrelevante el saber; como si lo único existente fuese la voluntad de poder. El acertado cuestionamiento de una supuesta angelical actividad científica por fuera de las mundanales disputas institucionalmente inscritas, de los constreñimientos y contingencias históricas, se reduce en algunas caricaturizaciones de Foucault a un argumento de que en últimas solo hay relaciones de poder. Esto es crucial, porque la interrupción genealógica no opera dentro de una celebratoria al relativismo epistémico.

El giro decolonial como estrategia de descolonización de la universidad apunta al cuestionamiento del profundo eurocentrismo que ha descalificado a los conocimientos de los sujetos coloniales. Esta estrategia pasa por evidenciar cómo este eurocentrismo es expresión de la colonialidad del saber que, a su vez, es la dimensión epistémica del sistema mundo moderno/colonial. Como lo han argumentado Catherine Walsh (2007) y Santiago Castro-Gómez (2007), la apuesta por interculturalizar o transculturalizar la universidad es central en el giro decolonial. Así, la estrategia del giro decolonial supone descentrar el monoculturalismo que ha definido no sólo los contenidos, sino también los términos-condiciones de la producción y apropiación del conocimiento, así como de la labor pedagógica y de su articulación social y política.

Ahora bien, Santiago Castro-Gómez (2007) es explícito en desmarcar esta estrategia de ciertos malentendidos que suelen presentarse cuando se cuestiona la hegemonía del eurocentrismo en la universidad: 
[...] la descolonización de la universidad, tal como aquí es propuesta, no conlleva una cruzada contra Occidente en nombre de algún tipo de autoctonismo latinoamericanista, de culturalismos etnocéntricos y de nacionalismos populistas, como suelen creer algunos. Tampoco se trata de ir en contra de la ciencia moderna y de promover un nuevo tipo de oscurantismo epistémico. Cuando decimos que es necesario ir "más allá" de las categorías de análisis y de las disciplinas modernas, no es porque haya que negarlas, ni porque éstas tengan que ser 'rebasadas' por algo 'mejor' (p. 90).

La tercera estrategia estaría dada por lo que hemos mencionado como provincializar a Europa. El término y la estrategia de provincializar a Europa han sido sugeridas por Dipesh Chakrabarty en un libro que ha sido traducido al castellano con el título Al margen de Europa. Para Chakrabarty (2008), provincializar a Europa significa desnaturalizar las narrativas eurocentradas evidenciando la parroquialidad e historicidad de modalidades de pensamiento, de ordenamientos políticos y de prácticas sociales que se esgrimen como universales.

Esto no significa que el proyecto de provincializar a Europa conduzca a un relativismo cultural ni, mucho menos, a un nacionalismo o nativismo no-europeo (Chakrabarty, 2008, p. 43). La idea no es simplemente rechazar a la modernidad, los universales, la ciencia o la razón arguyendo que son culturalmente específicos a Europa y, en consecuencia, intrínsecamente reproductores del eurocentrismo. Aunque esto último no deja de ser cierto, provincializar a Europa significa más bien desplazar a Europa del centro de la imaginación histórica, epistémica y política.

El centramiento de Europa que se reproduce al tomar como referentes privilegiados a los autores, conceptualizaciones y estilos académicos euro-estadounidenses, requiere ser revertido por un giro en los términos de la conversación, al igual que en las genealogías intelectuales e interlocutores que nos interpelan y constituyen. Esto no supone dejar de conversar con estos autores, ni de desconocer estas conceptualizaciones y estilos, sino complejizarlos, complementarlos 
e interrumpirlos ampliando nuestro espectro de conversaciones, hay que tomar en serio interlocutores que conversan en otros lenguajes, que suponen diferentes estilos intelectuales.

\section{Referencias}

Castro-Gómez, S. (2007). "Decolonizar la universidad. La hybris del punto cero y el diálogo de saberes". En: Ramón Grosfoguel y Santiago Castro-Gómez (eds.), El giro decolonial. Reflexiones para una diversidad epistémica más allá del capitalismo global, pp. 79-91. Bogotá: Siglo del Hombre Editores.

Césaire, A. (2006). Discurso sobre el colonialismo. Madrid: Akal.

Chakrabarty, D. (2008). Al margen de Europa: pensamiento poscolonial y diferencia histórica. Barcelona: Tusquets.

Escobar, A. (2003). Mundos y conocimientos de otro modo: el programa de investigación de modernidad/colonialidad Latinoamericano. Tabula Rasa (1): 51-86.

Foucault, M. (2001) Defender la sociedad. Buenos Aires: Fondo de Cultura Económica.

Kuwayama, T. (2004). Native Anthropology. The Japanese Challenge to Western Academic Hegemony. Melbourne: Trans Pacific Press.

Mato, D. (2002). "Estudios y otras prácticas latinoamericanas en cultura y poder", Estudios y otras prácticas latinoamericanas en cultura y poder. Buenos Aires: Clacso.

Mignolo, W. (2007). "El pensamiento decolonial: desprendimiento y apertura. Un manifiesto". En: Ramón Grosfoguel y Santiago Castro-Gómez (eds.), El giro decolonial. Reflexiones para una diversidad epistémica más allá del capitalismo global. pp. 2546. Bogotá: Siglo del Hombre Editores.

Quijano, A. 2000. Colonialidad del poder y clasificación social. Journal of World-System Research (2): 342-386.

Restrepo, E., \& Rojas, A. (2010). Inflexión decolonial: fuentes, conceptos y cuestionamientos. Popayán: Editorial Universidad del Cauca. 
Lins Ribeiro, G., \& Escobar, A. (2008). Antropologías del mundo: Transformaciones disciplinarias dentro de sistemas de poder. Popayán: Ciesas-Envión Editores.

De Sousa Santos, B. (2009). Una epistemología del sur: la reinvención del conocimiento y la emancipación social. México; Buenos Aires: Siglo XXI-Clacso.

Trouillot, M-R. (2011). Transformaciones globales. La antropología y el mundo moderno. Popayán: Editorial de la Universidad del Cauca.

Wallerstein, I. (2006). El capitalismo histórico. Buenos Aires: Siglo XXI.

Walsh, C. (2007). ¿Son posibles unas ciencias sociales/culturales otras? Reflexiones en torno a las epistemologías decoloniales. Nómadas. (26): 102-113.

Walsh, C. (2005). "Interculturalidad, colonialidad y educación", Ponencia en el Primer Seminario Internacional "(Etno)educación, multiculturalismo e interculturalidad”. Universidad del Cauca, 1 al 4 de noviembre. 